\title{
The role of adatoms in chloride-activated colloidal silver nanoparticles for surface-enhanced Raman scattering enhancement
}

\author{
Nicolae Leopold ${ }^{* 1}$, Andrei Stefancu ${ }^{* 1}$, Krisztian Herman ${ }^{1}$, István Sz. Tódor ${ }^{* 1}$, \\ Stefania D. lancu ${ }^{1}$, Vlad Moisoiu ${ }^{1}$ and Loredana F. Leopold ${ }^{*}$
}

Full Research Paper

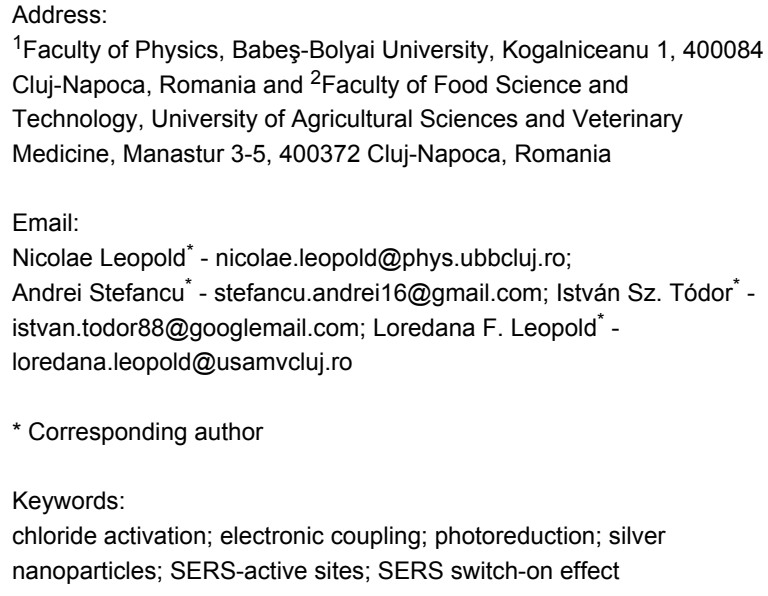

${ }^{1}$ Faculty of Physics, Babeş-Bolyai University, Kogalniceanu 1, 400084 Cluj-Napoca, Romania and ${ }^{2}$ Faculty of Food Science and

Technology, University of Agricultural Sciences and Veterinary

Medicine, Manastur 3-5, 400372 Cluj-Napoca, Romania

Email:

Nicolae Leopold* - nicolae.leopold@phys.ubbcluj.ro;

Andrei Stefancu* - stefancu.andrei16@gmail.com; István Sz. Tódor * -

istvan.todor88@googlemail.com; Loredana F. Leopold ${ }^{*}$ -

loredana.leopold@usamvcluj.ro

${ }^{*}$ Corresponding author

Keywords:

chloride activation; electronic coupling; photoreduction; silver

nanoparticles; SERS-active sites; SERS switch-on effect

Beilstein J. Nanotechnol. 2018, 9, 2236-2247.

doi:10.3762/bjnano.9.208

Received: 09 May 2018

Accepted: 03 August 2018

Published: 22 August 2018

Associate Editor: S. R. Cohen

(c) 2018 Leopold et al.; licensee Beilstein-Institut.

License and terms: see end of document.

\begin{abstract}
Chloride-capped silver nanoparticles (Cl-AgNPs) allow for high-intensity surface-enhanced Raman scattering (SERS) spectra of cationic molecules to be obtained (even at nanomolar concentration) and may also play a key role in understanding some fundamental principles behind SERS. In this study, we describe a fast $(<10 \mathrm{~min})$ and simple protocol for obtaining highly SERS-active colloidal silver nanoparticles (AgNPs) with a mean diameter of $36 \mathrm{~nm}$ by photoconversion from $\mathrm{AgCl}$ precursor microparticles in the absence of any organic reducing or capping agent. The resulting AgNPs are already SERS-activated by the $\mathrm{Cl}^{-}$ions chemisorbed onto the metal surface where the chloride concentration in the colloidal solution is $10^{-2} \mathrm{M}$. Consequently, the enhanced SERS spectra of cationic dyes (e.g., crystal violet or 9-aminoacridine) demonstrate the advantages of Cl-AgNPs compared to the as-synthesized AgNPs obtained by standard $\mathrm{Ag}^{+}$reduction with hydroxylamine (hya-AgNPS) or citrate (cit-AgNPs). The results of SERS experiments on anionic and cationic test molecules comparing Cl-AgNPs, hya-AgNPs and cit-AgNPs colloids activated with different amounts of $\mathrm{Cl}^{-}$and/or cations such as $\mathrm{Ag}^{+}, \mathrm{Mg}^{2+}$ or $\mathrm{Ca}^{2+}$ can be explained within the understanding of the adatom model - the chemisorption of cationic analytes onto the metal surface is mediated by the $\mathrm{Cl}^{-}$ions, whereas ions like $\mathrm{Ag}^{+}, \mathrm{Mg}^{2+}$ or $\mathrm{Ca}^{2+}$ mediate the electronic coupling of anionic species to the silver metal surface. Moreover, the SERS effect is switched on only after the electronic coupling of the adsorbate to the silver surface at SERS-active sites. The experiments presented in this study highlight the SERS-activating role played by ions such as $\mathrm{Cl}^{-}, \mathrm{Ag}^{+}, \mathrm{Mg}^{2+}$ or $\mathrm{Ca}^{2+}$, which is a process that seems to prevail over the Raman enhancement due to nanoparticle aggregation.
\end{abstract}




\section{Introduction}

The most common surface-enhanced Raman scattering (SERS) substrate is the silver colloid obtained by $\mathrm{Ag}^{+}$reduction with citrate (cit-AgNPs), proposed by Lee and Meisel in 1982 [1]. Although the preparation of cit-AgNPs requires boiling conditions, this colloid is often preferred due to its high preparation success rate. Another often used SERS substrate is the silver colloid obtained by $\mathrm{Ag}^{+}$reduction with sodium borohydride [2], or, more recently, with hydroxylamine hydrochloride (hyaAgNPs) [3]. As we will show in the present study, the higher Raman enhancement of the hya-AgNPs compared to as-synthesized cit-AgNPs arises from the presence of chemisorbed $\mathrm{Cl}^{-}$ions, which form SERS-active sites on the silver nanoparticle (AgNP) surface. More precisely, the as-synthesized hyaAgNP colloidal solution already contains $\mathrm{Cl}^{-}$ions in a concentration of $2.4 \mathrm{mM}$ due to the addition of the hydrochloride form of hydroxylamine to the reaction mixture, from which a small part of $\mathrm{Cl}^{-}$ions become chemisorbed onto the AgNP surface, thus generating the SERS-active sites.

Silver halides are a class of light-sensitive materials that have been extensively used for photographic films. Gao et al. [4] were the first to report SERS spectra acquired from a dye adsorbed onto silver nanoparticles formed at the surface of silver chloride particles suspended in water. The photoconversion of silver salts to metallic silver particles is the basic principle of film photography: silver ions in silver halide particles are photoreduced by the transfer of electrons from halide ions $[4,5]$.

Chloride anions are often added to metal colloids to enhance the Raman signal of the analytes. This phenomenon can be explained by two distinct mechanisms: the electromagnetic mechanism and the chemical effect. Each mechanism is believed to contribute complementary to the Raman scattering enhancement of the adsorbate in SERS spectroscopy. When the SERS effect is explained in terms of the electromagnetic mechanism, an aggregating effect of the colloidal nanoparticles is attributed to the chloride ions [6], whereas in the chemical mechanism, a so-called activation effect is attributed to the chloride ions [7].

The electromagnetic mechanism arises due to enhanced local optical fields at the site of the molecule situated in the close proximity of the metal surface. This local enhancement is determined by the resonant excitation of surface plasmons, which are collective oscillations of the conducting electrons in the metallic nanoparticles (also called surface plasmon resonances) [8]. Although this model does not require a chemical contact, the magnitude of the electromagnetic field outside the particle decreases with the third power of radial distance [9]. This means that the field enhancement of Raman scattering decreases strongly with the distance from the metal surface.

Chloride ions, when used at concentrations higher than $0.1 \mathrm{M}$, induce the aggregation of the metal colloids. The addition of $\mathrm{Cl}^{-}$ions to the colloidal solution reduces the absolute zeta potential value of the nanoparticles, leading to a decrease in the stability of the colloid and therefore the aggregation of nanoparticles. Consequently, according to literature, electromagnetic hot spots, i.e., sites of highly increased field strength, are generated in the gaps between adjacent nanoparticles [10,11]. The maximal values for electromagnetic enhancement are on the order of $10^{6}-10^{7}$ for isolated metal nanoparticles [8].

The chemical mechanism of Raman signal enhancement is based on a metal-molecule electronic transition [8,12]. The electronic coupling of the molecule with the metal surface of the nanoparticle leads to a significant increase in the Raman cross-section of the adsorbed molecule. The chargetransfer electronic transition can be explained as follows $[8,12-$ 14]:

$$
\begin{aligned}
& \mathrm{M}-\mathrm{A}_{(\mathrm{v}=0)}+h v_{\text {laser }} \rightarrow \\
& \mathrm{M}^{+}-\mathrm{A}_{(\text {LUMO occupied })}^{-} \rightarrow \\
& \mathrm{M}-\mathrm{A}_{(\mathrm{v}=1)}+h v_{\text {Stokes }}
\end{aligned}
$$

The chemical mechanism of SERS involves the absorption of a photon and the excitation of an electron from the Fermi level of the metallic nanoparticle (M) to the LUMO orbital of the adsorbed molecule (A). Thus, an excited state is achieved $\left(\mathrm{M}^{+}-\mathrm{A}^{-}\right)$. If the molecule remains vibrationally excited during the transition of the electron from the excited state of the adsorbed molecule back to the metallic nanoparticle, then the transition is accompanied by the emission of a Raman photon $\left(h v_{\text {Stokes }}\right)$.

As proposed by Otto and co-workers [12,13], adsorbed atoms (adatoms) facilitate the metal-molecule electronic transfer, providing pathways for these transitions. Thus, according to the adatom model of SERS, the Raman enhancement effect appears only if the adsorbed molecule is bound to an adatom $[13,15,16]$. The adsorption of chloride ions will lead to the formation of additional adatoms on the surface of the nanoparticles, thus activating the metal surface of the nanoparticle $[12,13,17]$.

Likewise, the formation of complexes of the type $\mathrm{Ag}^{+}$-phthalazine-halide anion on AgNP surfaces has also been proposed by Muniz-Miranda and Sbrana [18]. 
In contrast to the electromagnetic mechanism, the chemical effect requires an electronic contact between adsorbate and metal surface [14]. The enhancement factor for the chemical effect is estimated to be between 10-100 [8].

In this study, we describe the synthesis of a stable colloidal solution of AgNPs capped only with $\mathrm{Cl}^{-}$ions, where the chemisorbed part of the $\mathrm{Cl}^{-}$ions forms the SERS-active sites for cationic molecules. Furthermore, the addition of cations such as $\mathrm{Ag}^{+}, \mathrm{Mg}^{2+}$ or $\mathrm{Ca}^{2+}$ increases the number of SERS-active sites on the AgNP surface by mediating the chemisorption of $\mathrm{Cl}^{-}$ions. Hence, cationic dye molecules are electronically coupled to the silver surface at these SERS-active sites, leading to high-intensity SERS spectra. The appearance of the SERS effect only after the electronic coupling of the analyte to the Ag surface explains why the citrate surfactant is not SERS active in the as-synthesized cit-AgNPs. Nevertheless, the SERS spectrum of the citrate surfactant can be turned on by the chemisorption of the citrate molecules onto the metal surface, mediated by cations such as $\mathrm{Ag}^{+}, \mathrm{Mg}^{2+}$ or $\mathrm{Ca}^{2+}$. In general, all the experiments performed in this study prove that the SERS effect is turned on only after the electronic coupling of the analyte to the Ag surface at SERS-active sites.

\section{Results and Discussion $\mathrm{Cl-AgNP}$ synthesis monitoring}

Cl-AgNPs can be synthesized within 10 min of light exposure. Figure 1 shows a picture of the experimental setup used for the synthesis of Cl-AgNPs (Figure 1A) and a picture of the resulting colloid (Figure 1B).

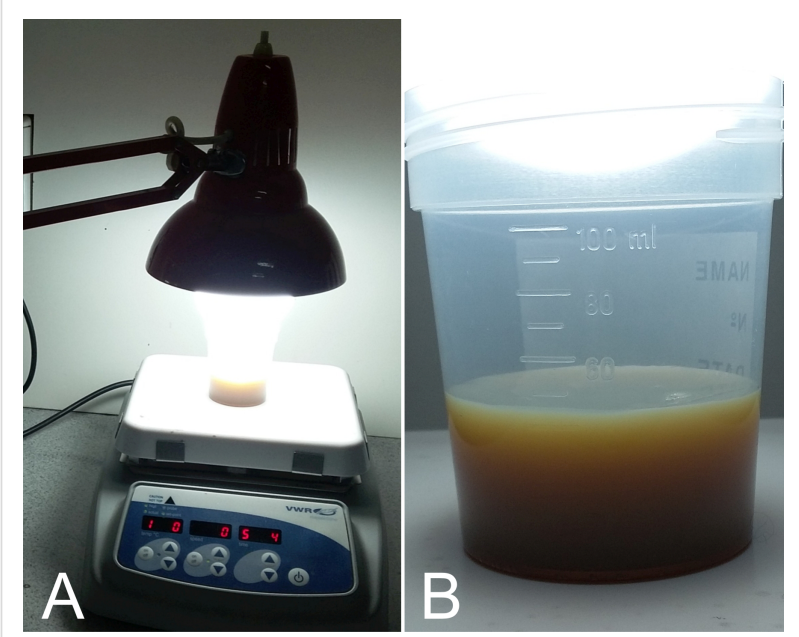

Figure 1: (A) Picture showing the experimental arrangement for the synthesis of Cl-AgNPs. A desk lamp with an LED bulb was placed above the beaker containing the reaction mixture. (B) Picture of the colloidal solution of $\mathrm{Cl}-\mathrm{AgNPs}$ obtained after $10 \mathrm{~min}$ of light exposure.
After the first $3 \mathrm{~min}$ of light exposure, the solution turned pale yellow; after 5 min of light exposure, an intense gray-brown color indicated a high concentration of AgNPs in the solution. Also, at this time, gaseous reaction products were observed, probably due to the $\mathrm{O}_{2}$ released during the decomposition of hydrogen peroxide. No further color changes could be visually observed after $5 \mathrm{~min}$ of light exposure, but the reaction mixture was further exposed to light for a total of $15 \mathrm{~min}$ and afterwards stored in the dark for $24 \mathrm{~h}$.

A slightly faster synthesis was observed when covering the beaker in aluminum foil, due to the local increase in light intensity. Also, we observed that a LED bulb with a "cool light" emission spectrum led to a faster synthesis compared to an equivalent power "warm light" emitting bulb. Nonetheless, similar results were obtained when using a lower power LED bulb (12 W, $6400 \mathrm{~K}$, cool white, $960 \mathrm{~lm}$ ).

However, in most of the reported studies on silver nanoparticles synthesized by photoreduction, the photosynthesis was performed in the presence of organic stabilizing agents such as citrate [19] or poly ( $N$-vinylpyrrolidone) [20,21]. The photoreduction of $\mathrm{AgCl}$ crystals has also been previously reported; however, the photoconversion of $\mathrm{AgCl}$ to silver nanoparticles was performed in the presence of stabilizing agents, such as proteins [22] or DNA $[23,24]$. Compared to these studies, the most important novelty of our protocol is represented by the rapid synthesis in addition to the absence of any organic capping agent.

The UV-vis spectral monitoring of the Cl-AgNPs during the synthesis process (Figure 2) shows the photoconversion of $\mathrm{AgCl}$ microparticles to AgNPs in the first $10 \mathrm{~min}$ of light exposure.

Mixing the silver nitrate and sodium chloride in the reacting solution resulted in the generation of a flocculent precipitate of $\mathrm{AgCl}$. The presence of $\mathrm{AgCl}$ particles in the solution was evidenced in the UV-vis spectra as an intense absorption band at $254 \mathrm{~nm}$ (Figure 2) [25,26]. It should be mentioned that none of the reagents measured separately showed an absorption band at this wavelength and that the $\mathrm{AgCl}$ absorption band can also be observed in the UV-vis spectrum of a mixture of only $\mathrm{AgNO}_{3}$ and $\mathrm{NaCl}$ (Supporting Information File 1, Figure $\mathrm{S} 1$ ).

During the exposure of the reaction mixture to light, the intensity of the $254 \mathrm{~nm}$ band due to $\mathrm{AgCl}$ absorption decreases, while the plasmonic band intensity of the nascent AgNPs increases. The UV-vis spectrum recorded after 1 min shows a very low intensity plasmonic band at $419 \mathrm{~nm}$, indicating the formation of 


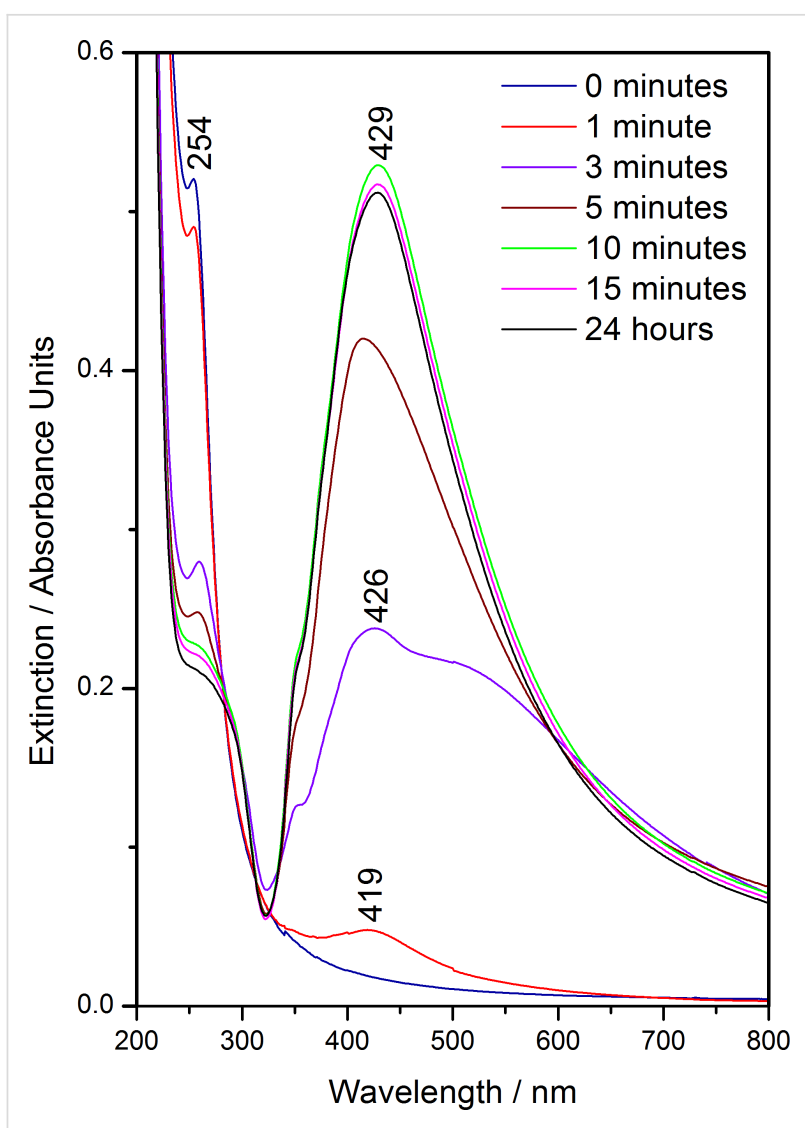

Figure 2: UV-vis extinction spectra obtained during the Cl-AgNP synthesis, recorded after $0,1,3,5,10$ and 15 min of light exposure to the reacting mixture in addition to $24 \mathrm{~h}$ after the colloid synthesis.

only a small number of metallic silver nanoparticles, as well as a slight decrease in the intensity of the $\mathrm{AgCl}$ band.

After 3 min of light exposure, the UV-vis absorption spectrum of the reaction mixture shows a large plasmonic band with a maximum at $426 \mathrm{~nm}$ and a wide shoulder around $550 \mathrm{~nm}$, indicating a high polydispersity of the formed silver nanostructures. Simultaneously, a decrease in the intensity of the $\mathrm{AgCl}$ absorption band at $254 \mathrm{~nm}$ is observed, whereby the intensity of the $\mathrm{AgCl}$ band is then comparable to that of the plasmonic band. However, after $5 \mathrm{~min}$ of light exposure, the plasmon resonance band became narrower, showing a typical shape for silver colloids.

Between 5 and 10 min of light exposure, the intensity of the plasmonic band at $429 \mathrm{~nm}$ reaches its maximum, and no further increase of the plasmonic band intensity could be observed between 10 and $15 \mathrm{~min}$ of light exposure. Furthermore, within $24 \mathrm{~h}$ from the synthesis of the colloid, no notable changes in the UV-vis spectrum were observed. Consequently, we can conclude that the colloidal solution is synthesized within the first 10 min of light exposure.
Intense light exposure is critical for the synthesis of Cl-AgNPs, since the reaction does not proceed in the absence of an intense light source, regardless of the amount of hydrogen peroxide added in the reaction mixture.

It should be mentioned that the formation of stable, SERSactive Cl-AgNP colloids can also be obtained in the absence of hydrogen peroxide, but in this case, the complete synthesis of the silver colloids takes several hours. The UV-vis absorption monitoring of the Cl-AgNP synthesis obtained in the absence of hydrogen peroxide is presented in Supporting Information File 1, Figure S2A, whereas the SERS activity of the Cl-AgNPs tested after different times of light exposure is shown in Figure S2B (using crystal violet as an analyte).

The formation of $\mathrm{AgNPs}$ from $\mathrm{AgCl}$ precursor microparticles can be also followed in the scanning electron microscopy (SEM) micrographs depicted in Figure 3.

Figure 3a shows 1-2 $\mu \mathrm{m} \mathrm{AgCl}$ particles formed after mixing silver nitrate and sodium chloride in the reacting solution.

During the photoreduction process, the surface of the $\mathrm{AgCl}$ particles becomes covered with clusters of Ag atoms, which was also reported by Wang et al. [27].

The silver atom clusters grow continuously during light exposure, leading to the formation of AgNPs on the surface of $\mathrm{AgCl}$ microparticles, as shown in Figure 3b-d. Figure 3e,f shows the AgNPs obtained after the full photoconversion from $\mathrm{AgCl}$ precursor microparticles, where the majority of the Cl-AgNPs in Figure $3 \mathrm{f}$ have a diameter between 30 and $40 \mathrm{~nm}$. A mean diameter of $36 \mathrm{~nm}$ was estimated from the electron microscopy micrographs.

\section{SERS spectra of cations}

To highlight the role of the $\mathrm{Cl}^{-}$ions in the generation of SERSactive sites on the silver surface and chemisorption of cationic analyte molecules, we performed comparative SERS experiments using Cl-AgNPs, cit-AgNPs and hya-AgNPs. Crystal violet chloride and 9-aminoacridine hydrochloride monohydrate were used as test analytes. Figure 4 shows comparatively the SERS spectra of the two cationic dyes obtained using the three silver colloids.

By far, the Cl-AgNPs displayed the highest SERS intensity for both cationic dyes, followed by hya-AgNPs, which showed only low intensity SERS bands, and finally cit-AgNPs, for which no SERS bands were visible. The higher SERS enhancement for the Cl-AgNPs was expected, since this colloid contains around 4 times more $\mathrm{Cl}^{-}$ions $(10 \mathrm{mM})$ compared to hya-AgNPs 

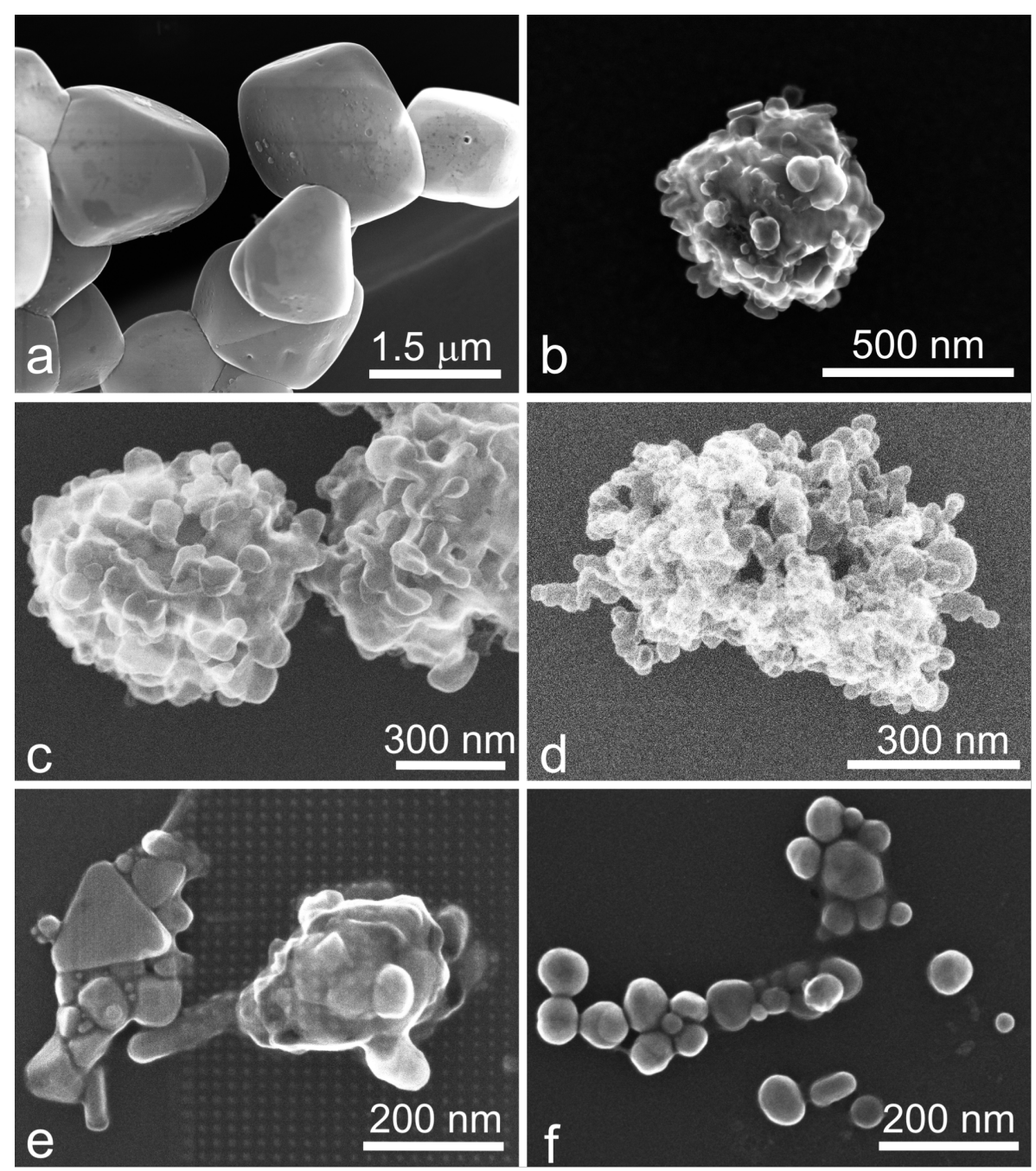

Figure 3: SEM micrographs showing different stages during the photoconversion of $\mathrm{AgCl}$ microparticles (a) to AgNPs (f).

(2.4 $\mathrm{mM})$. However, the SERS enhancement of Cl-AgNPs is more than one order of magnitude higher than that of hyaAgNPs. This finding can be explained within the adatom model, which states that the enhancement is not due to the "free" $\mathrm{Cl}^{-}$ions, but only due to chemisorbed $\mathrm{Cl}^{-}$ions (adatoms), which form SERS-active sites on the AgNPs. Hence, the chemisorbed $\mathrm{Cl}^{-}$ions mediate the electronic coupling of the cationic molecules by forming $\mathrm{Cl}^{-}$-adsorbate surface complexes [18]. Consequently, the fact that the highest Raman enhancement was obtained using Cl-AgNPs as substrate can be explained by the greater number of SERS-active sites in the case of this colloid. The SERS activation effect of chloride was reported in several studies. Futamata and Maruyama showed that $\mathrm{Cl}^{-}$ions induce the electronic interaction between silver nanoparticles and rhodamine 6G (R6G), leading to intense SERS spectra [28]. Doering and Nie highlighted the activating effect of halide ions such as $\mathrm{Cl}^{-}, \mathrm{Br}^{-}$or $\mathrm{I}^{-}$on single-particle SERS [29]. In addition, they observed that the selective removal of $\mathrm{Ag}^{+}$by thiosulfate (a fixative used in black-and-white photography to dissolve residual $\mathrm{Ag}^{+}$cations) leads to a dramatic decrease in the number of SERS-active nanoparticles, underlining thus the need for $\mathrm{Ag}^{+}$cations for the generation of SERS-active sites on the nanoparticle surface [28,29].

When using the cit-AgNPs as a SERS substrate, no SERS spectra of the dye molecules could be obtained since the Lee-Meisel colloid does not contain any chloride ions that could form SERS-active sites. Small amounts of $\mathrm{Cl}^{-}$ions $\left(10^{-8}-10^{-7} \mathrm{M}\right)$ are nonetheless present in the solution, since the test molecules were added as chloride salts, but not enough to observe a SERS effect.

However, after adding $0.1 \mathrm{M} \mathrm{NaCl}$ to the cit-AgNPs or hyaAgNPs, intense SERS spectra of crystal violet or 9-aminoacri- 

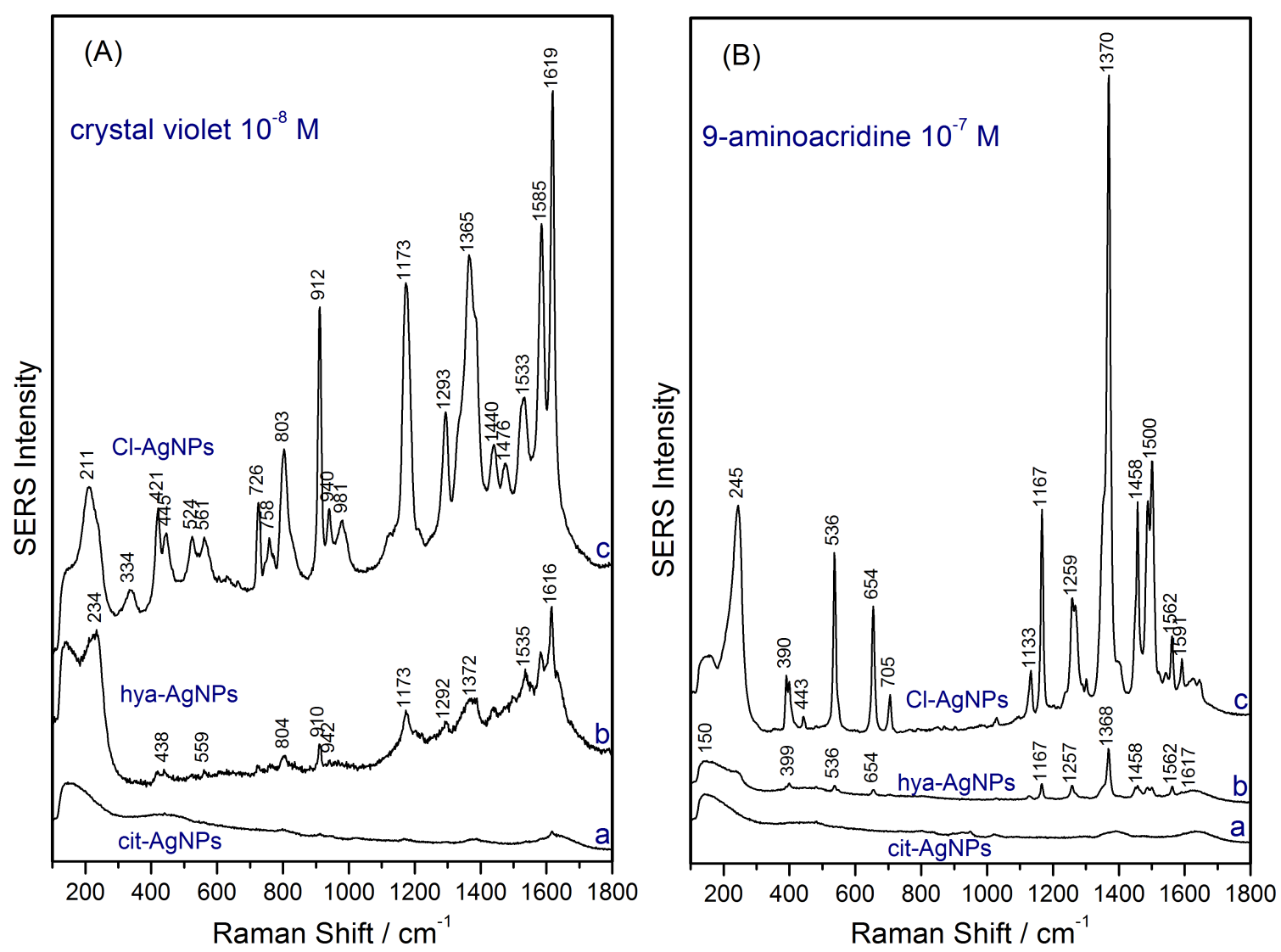

Figure 4: (A) SERS spectra of crystal violet $10^{-8} \mathrm{M}$ and (B) 9-aminoacridine $10^{-7} \mathrm{M}$ obtained using three different silver colloidal solutions as substrate: cit-AgNPs (a), hya-AgNPs (b), and Cl-AgNPs (c), as indicated in the figure.

dine were obtained (data not shown). We suppose that besides aggregating the colloid upon $\mathrm{Cl}^{-}$addition, the increase in the SERS intensity of the test analytes is also explained by the generation of more SERS-active sites on the silver surface of the cit-AgNPs or hya-AgNPs.

In the next section we will show that cations such as $\mathrm{Ag}^{+}, \mathrm{Mg}^{2+}$ or $\mathrm{Ca}^{2+}$ promote the chemisorption of $\mathrm{Cl}^{-}$ions, thereby generating SERS-active sites for cationic dye molecules. Thus, instead of adding additional $\mathrm{Cl}^{-}$ions, the SERS intensity of the analyte can be increased by adding cations such as $\mathrm{Mg}^{2+}$ or $\mathrm{Ca}^{2+}$, which promote the chemisorption of more $\mathrm{Cl}^{-}$onto the silver surface from the total amount of "free" $\mathrm{Cl}^{-}$ions present in the hya-AgNPs or Cl-AgNP colloids.

\section{AgNP activation for obtaining SERS spectra of anions and cations}

The Raman spectra of conventional silver colloids used as a SERS substrate contain no distinct peaks, since the concentration of the reagents (in the $\mathrm{mM}$ range) is too low for normal Raman spectroscopy. The surfactant (capping agent) plays a key role in the stabilization of the AgNPs by prohibiting aggre- gation via electrostatic repulsion between AgNPs. However, the surfactant surrounding the AgNPs does not exhibit a SERS spectrum if an electronic coupling with the silver surface is absent. Figure 5 shows the spectra of the Cl-AgNP and citAgNP colloids. For comparison, the Raman spectrum of water is given.

The blank SERS spectrum of Cl-AgNPs (Figure 5A) shows a very low intensity band at $242 \mathrm{~cm}^{-1}$ assigned to $\mathrm{Ag}-\mathrm{Cl}$ vibration, indicating that a small proportion of $\mathrm{Cl}^{-}$ions are chemisorbed onto the silver surface. These chemisorbed $\mathrm{Cl}^{-}$ions form the SERS-active sites on the as-synthesized colloidal AgNPs which facilitate the recording of intense SERS spectra of cationic dye analytes (Figure 4). In contrast, the blank spectrum of cit-AgNPs (Figure 5B) shows a similar spectral shape to that of water, i.e., there are no SERS bands to indicate an activation of the cit-AgNPs. Thus, as shown in Figure 4, the as-synthesized cit-AgNPs colloid is SERS inactive for crystal violet $10^{-8} \mathrm{M}$ and 9 -aminoacridine $10^{-7} \mathrm{M}$.

The number of SERS-active sites on Cl-AgNPs can be increased considerably by promoting the chemisorption of more 

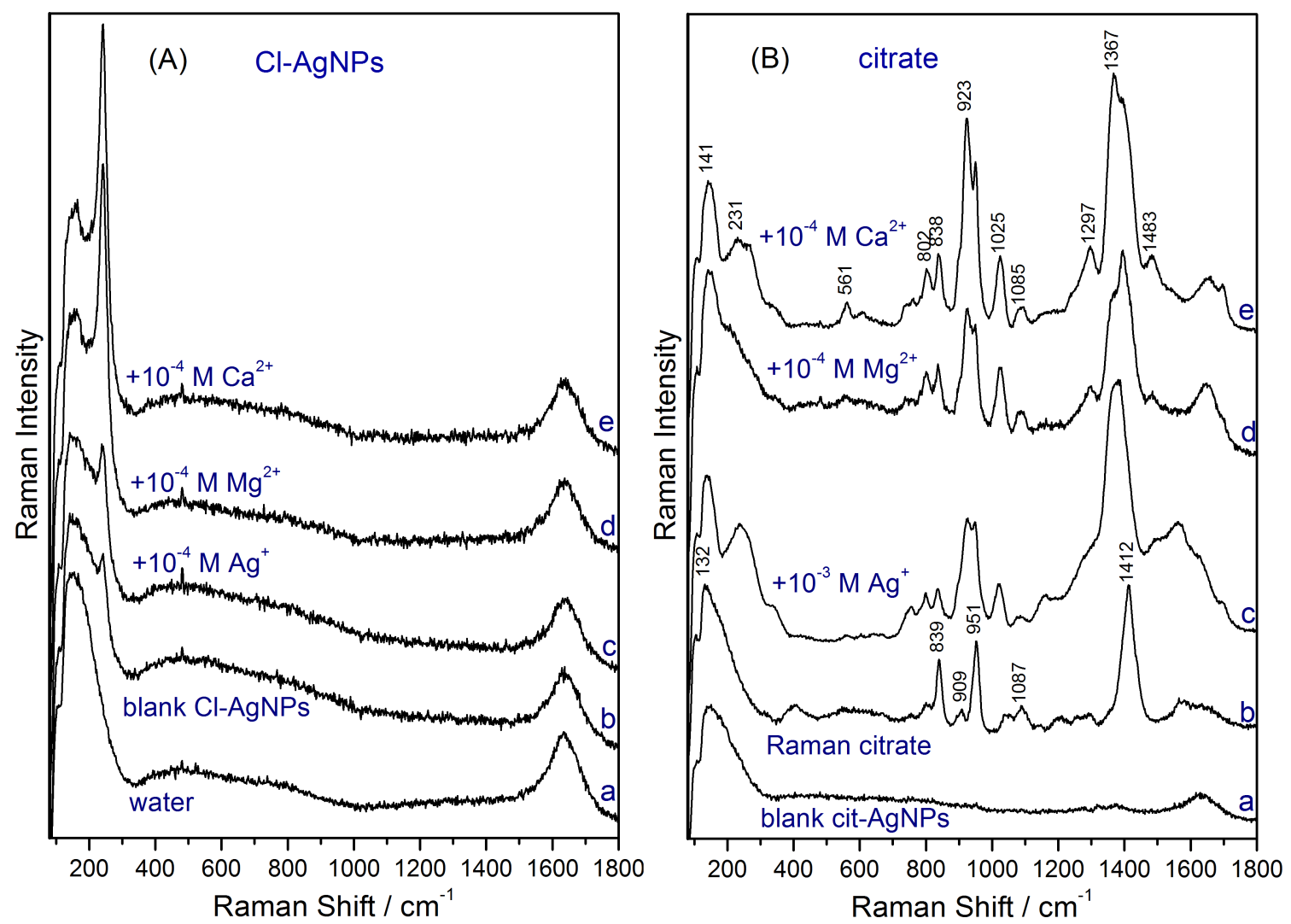

Figure 5: (A) Raman spectrum of water (a), SERS spectrum of Cl-AgNPs (b), SERS spectrum of Cl-AgNPs after addition of $10^{-4} \mathrm{M} \mathrm{AgNO}_{3}$ (c), $10^{-4} \mathrm{M} \mathrm{MgSO}_{4}(\mathrm{~d})$, and $10^{-4} \mathrm{M} \mathrm{Ca}\left(\mathrm{NO}_{3}\right)_{2}$ (e) showing the intense Ag-Cl SERS band. (B) Raman blank spectrum of cit-AgNPs (a), Raman spectrum of $1 \mathrm{M}$ citrate (b), SERS spectra of citrate obtained after addition of $10^{-3} \mathrm{M} \mathrm{AgNO}_{3}$ (c), $10^{-4} \mathrm{M} \mathrm{MgSO}_{4}(\mathrm{~d})$, and $10^{-4} \mathrm{M} \mathrm{Ca}_{\left(\mathrm{NO}_{3}\right)}(\mathrm{e})$ to the cit-AgNPs

$\mathrm{Cl}^{-}$ions onto the AgNP surface using cations such as $\mathrm{Ag}^{+}$, $\mathrm{Mg}^{2+}$ or $\mathrm{Ca}^{2+}$. Thus, by adding salts such as $\mathrm{AgNO}_{3}, \mathrm{MgSO}_{4}$ or $\mathrm{Ca}\left(\mathrm{NO}_{3}\right)_{2}$ at a concentration of $10^{-4} \mathrm{M}$ to the Cl-AgNPs, an increase in the intensity of the $\mathrm{Ag}-\mathrm{Cl}$ vibration band at $242 \mathrm{~cm}^{-1}$ is observed due to the additional chemisorption of $\mathrm{Cl}^{-}$ions onto the silver surface mediated by the $\mathrm{Ag}^{+}, \mathrm{Mg}^{2+}$ or $\mathrm{Ca}^{2+}$ (Figure $5 \mathrm{~A}$ ). $\mathrm{Ag}^{+} 10^{-4} \mathrm{M}$ ions induce only a slight increase of the number of SERS-active sites, as can be deduced from the slight intensity increase of the $242 \mathrm{~cm}^{-1}$ band. It should be mentioned that the Cl-AgNP colloid activated with $\mathrm{Ag}^{+}, \mathrm{Mg}^{2+}$ or $\mathrm{Ca}^{2+} 10^{-4} \mathrm{M}$ is stable for weeks.

In line with the adatom model, the generation of additional SERS-active sites on the metal surface leads to an increase in the SERS intensity of analytes, as shown for $10^{-9} \mathrm{M}$ crystal violet in Figure 6A. The addition of $1 \mathrm{mM} \mathrm{MgSO}_{4}$ or $\mathrm{Ca}\left(\mathrm{NO}_{3}\right)_{2}$ leads to a visibly increased SERS signal of crystal violet $10^{-9} \mathrm{M}$ (roughly three times). Besides the intense SERS bands of the analyte, the intense $\mathrm{Ag}-\mathrm{Cl}$ band at $242 \mathrm{~cm}^{-1}$ evidences the high number of SERS-active sites. However, in the case of
Cl-AgNPs, the increase in intensity after generating additional SERS-active sites on the Cl-AgNP surface is not as dramatic, since most of the analyte molecules were already chemisorbed in the as-synthesized colloidal solution, due to their low concentration $\left(10^{-9} \mathrm{M}\right)$.

The increase in the SERS intensity of $10^{-9} \mathrm{M}$ crystal violet is more impressive when using activated hya-AgNPs as a substrate, as shown in Figure 6B. The hya-AgNPs contain $\mathrm{Cl}^{-}$ions in a concentration of $2.4 \mathrm{mM}$ and the addition of cations such as $\mathrm{Mg}^{2+}$ or $\mathrm{Ca}^{2+}$ in concentrations of $1 \mathrm{mM}$ mediates the chemisorption of more chloride ions onto the silver surface and the formation of more SERS-active sites. Thus, the very low intensity SERS spectrum of $10^{-9} \mathrm{M}$ crystal violet, recorded with the as-synthesized hya-AgNPs, becomes a very intense SERS spectrum after the addition of $\mathrm{MgSO}_{4}$ or $\mathrm{Ca}\left(\mathrm{NO}_{3}\right)_{2} 1 \mathrm{mM}$ (Figure 6B).

The lack of SERS-active sites in the as-synthesized cit-AgNPs allows the observation in an instructive manner of the SERS 

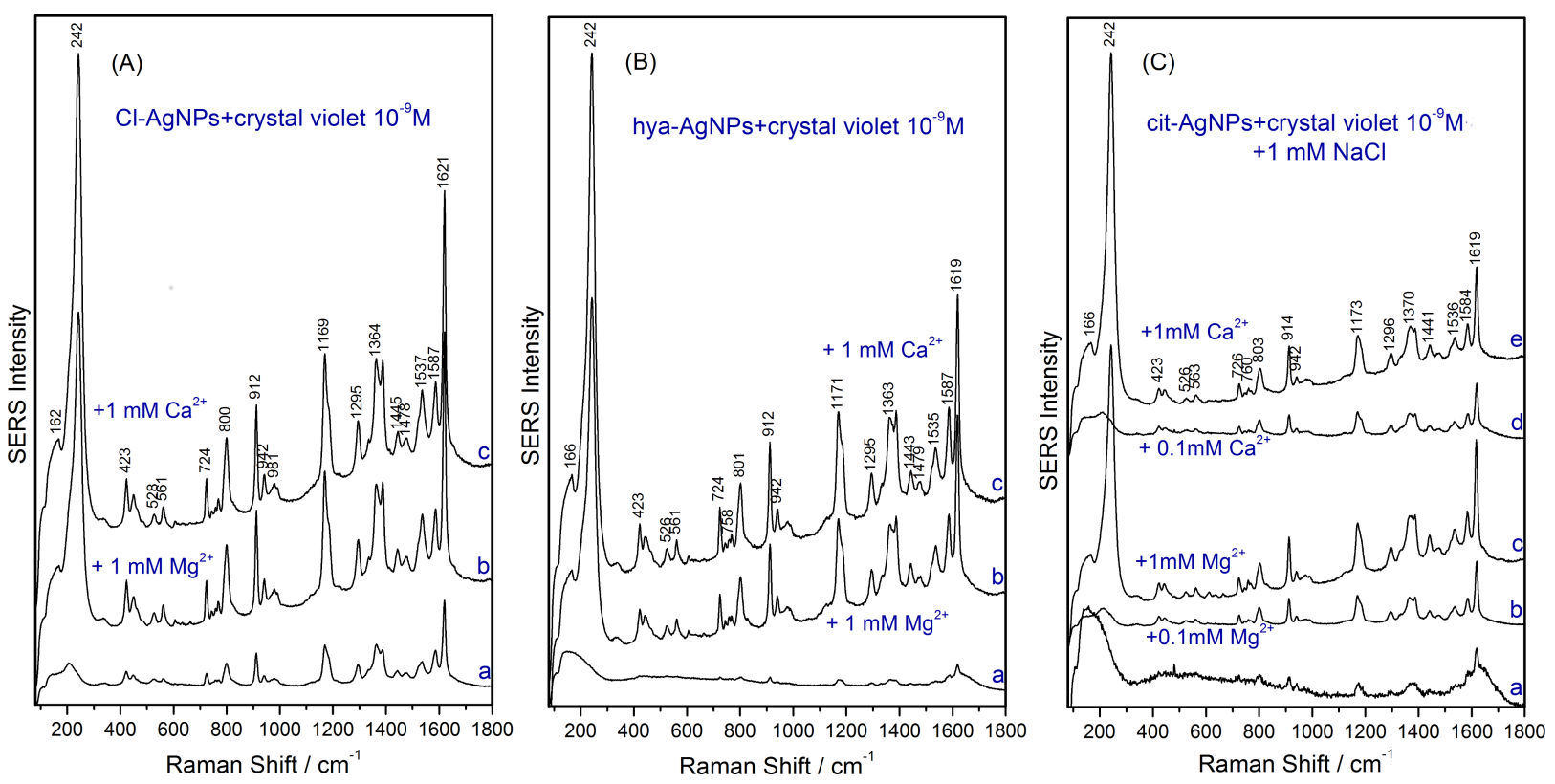

Figure 6: SERS spectra of $10^{-9} \mathrm{M}$ crystal violet recorded using the following as substrates: (A) $\mathrm{Cl}-\mathrm{AgNPs}(\mathrm{a}), \mathrm{Cl}-\mathrm{AgNPs}$ and $1 \mathrm{mM} \mathrm{MgSO}{ }_{4}$ (b), $1 \mathrm{mM} \mathrm{Ca}\left(\mathrm{NO}_{3}\right)_{2}$ (c); (B) hya-AgNPs (a), hya-AgNPs and $1 \mathrm{mM} \mathrm{MgSO}_{4}$ (b), $1 \mathrm{mM} \mathrm{Ca}\left(\mathrm{NO}_{3}\right)_{2}$ (c); (C) cit-AgNPs+1mM NaCl (a), cit-AgNPs+1mM NaCl and $10^{-4} \mathrm{M} \mathrm{MgSO}_{4}$ (b), cit-AgNPs+1 $\mathrm{mM} \mathrm{NaCl}$ and $1 \mathrm{mM} \mathrm{MgSO}_{4}$ (c), cit-AgNPs+1 $\mathrm{mM} \mathrm{NaCl}$ and $10^{-4} \mathrm{M} \mathrm{Ca}\left(\mathrm{NO}_{3}\right) 2(\mathrm{~d}), \mathrm{cit}_{-} \mathrm{AgNPs}+1 \mathrm{mM} \mathrm{NaCl}$ and $1 \mathrm{mM} \mathrm{Ca}\left(\mathrm{NO}_{3}\right)_{2}(\mathrm{e})$.

switch effect after SERS activation of colloid and the coupling of the analyte to the silver surface.

Since the as-synthesized cit-AgNPs did not allow the recording of SERS bands of $10^{-9} \mathrm{M}$ crystal violet, we added $1 \mathrm{mM} \mathrm{NaCl}$ to activate the cit-AgNPs. However, the presence of $1 \mathrm{mM} \mathrm{Cl}^{-}$ led only to a weak activation of the cit-AgNPs, where the SERS intensity of $10^{-9} \mathrm{M}$ crystal violet (Figure 6C) was comparable to that recorded with the as-synthesized hya-AgNPs (Figure 6B).

However, the further addition of $\mathrm{MgSO}_{4}$ or $\mathrm{Ca}\left(\mathrm{NO}_{3}\right)_{2}$ in concentrations ranging from $10^{-4}-10^{-3} \mathrm{M}$ to a cit- $\mathrm{AgNP}$ solution which already contains $1 \mathrm{mM} \mathrm{NaCl}$, led to the switching on of the SERS effect and the recording of very intense SERS spectra of $10^{-9} \mathrm{M}$ crystal violet. Accordingly, the intense SERS spectra of crystal violet obtained after the generation of SERSactive sites on the surface of cit-AgNPs by adding $10^{-3} \mathrm{M} \mathrm{Cl}^{-}$ and $10^{-4} \mathrm{M} \mathrm{Mg}^{2+}$ or $\mathrm{Ca}^{2+}$ are shown in Figure 6C. Moreover, a further raise in intensity of the SERS spectra of crystal violet can be observed by increasing the concentration of $\mathrm{Mg}^{2+}$ or $\mathrm{Ca}^{2+}$ ions to $1 \mathrm{mM}$ in the case of cit-AgNPs which were previously activated by adding $1 \mathrm{mM} \mathrm{NaCl}$ (Figure 6C).

In summary, high-intensity SERS spectra of cationic dyes can be recorded with all three silver colloids after activation. For this, our recommendation is that the silver colloid should contain $\mathrm{Cl}^{-}$anions in a concentration range between $10^{-3}-10^{-2} \mathrm{M}$ and $\mathrm{Mg}^{2+}$ or $\mathrm{Ca}^{2+}$ in concentrations between $10^{-4}-10^{-3} \mathrm{M}$.

In order to study the effect of the $\mathrm{Cl}^{-}$surface density and dye concentration on Raman enhancement, we performed a series of SERS studies on R6G in the $10^{-7}-10^{-11} \mathrm{M}$ concentration range using Cl-AgNPs and cit-AgNPs, the details of which are included in Supporting Information File 1 (Figures S3 and S4).

In the case of R6G analyzed with as-synthesized Cl-AgNPs, the limit of detection was $10^{-8} \mathrm{M}$ (Supporting Information File 1, Figure S3A, spectrum b). In other words, the number of $\mathrm{Cl}^{-}$SERS-active sites on the silver surface is enough to observe the SERS spectrum of R6G down to a concentration of $10^{-8} \mathrm{M}$. However, the detection limit of R6G can be reduced to $10^{-11} \mathrm{M}$ by adding $\mathrm{Ca}^{2+}$ ions, which promote the adsorption of additional $\mathrm{Cl}^{-}$and thus increase the number of $\mathrm{Cl}^{-}$SERS-active sites (Supporting Information File 1, Figure S3D, spectrum b and c). Although the absolute surface density of $\mathrm{Cl}^{-}$is difficult to calculate, the relative surface density of $\mathrm{Cl}^{-}$can be estimated based on the $240 \mathrm{~cm}^{-1}$ band of $\mathrm{Ag}-\mathrm{Cl}$. For example, when analyzing $10^{-11} \mathrm{M}$ R6G with $\mathrm{Cl}-\mathrm{AgNPs}$, the results show that the intensity of the $240 \mathrm{~cm}^{-1}$ band increases from $\approx 50$ counts when using $0.1 \mathrm{mM} \mathrm{Ca}^{2+}$ to about $\approx 250$ counts for $1 \mathrm{mM} \mathrm{Ca}^{2+}$ (Supporting Information File 1, Figure S3D, spectrum $b$ and $c)$. 
By increasing the $\mathrm{Ca}^{2+}$ concentration from $0.1 \mathrm{mM}$ up to $1 \mathrm{mM}$ in Cl-AgNPs, an increase of the number of SERS-active sites in the Cl-AgNPs was observed, as highlighted in Supporting Information File 1, Figure S3 by the increase of the intensity of the R6G SERS spectra (Figure S3A,B) or of the $\mathrm{Ag}-\mathrm{Cl}$ band intensity (Figure S3C,D). Further increasing the $\mathrm{Ca}^{2+}$ concentration leads to the aggregation of the colloid and therefore the SERS spectrum of the analyte is not observable anymore.

In the case of $10^{-7}, 10^{-8}$ and $10^{-11} \mathrm{M}$ R6G analyzed with as-synthesized cit-AgNPs, only the fluorescence emission due to R6G was observed (Supporting Information File 1, Figure $\mathrm{S} 4 \mathrm{~A}, \mathrm{~B})$. The SERS spectrum of R6G $10^{-8} \mathrm{M}$ shows only fluorescence emission even after the addition of $10^{-4} \mathrm{M} \mathrm{Ca}^{2+}$ (Supporting Information File 1, Figure S4A, spectrum b). However, after the addition of $10^{-3} \mathrm{M} \mathrm{Cl}^{-}$ions, the SERS spectrum of R6G is turned on (Supporting Information File 1, Figure S4A, spectrum c). An increase in the intensity of the SERS spectrum of R6G is observed after increasing the $\mathrm{Cl}^{-}$concentration to $10 \mathrm{mM}$ (Supporting Information File 1, Figure S4A, spectrum d), thus evidencing the increase in $\mathrm{Cl}^{-}$SERS-active sites.

A detection limit of $10^{-11} \mathrm{M}$ for R6G was obtained also with cit-AgNPs. However, an activation of the cit-AgNPs with $0.1 \mathrm{mM} \mathrm{Ca}\left(\mathrm{NO}_{3}\right)_{2}$ and $10 \mathrm{mM} \mathrm{NaCl}$ was necessary (Supporting Information File 1, Figure S4B, spectrum b).

Figure S4 in Supporting Information File 1 highlights an increase of SERS-active sites by increasing the $\mathrm{Cl}^{-}$concentration up to $10 \mathrm{mM}$, clearly showing that the SERS intensity is proportional to the number of generated $\mathrm{Cl}^{-}$adatoms up to a concentration of $10^{-2} \mathrm{M}$. Further increasing the $\mathrm{Cl}^{-}$concentration leads to the aggregation of the colloid and therefore the SERS spectrum of the analyte is not observable anymore.

Therefore, these results suggest that the number of active SERS sites is the key in determining the enhancement factor and that nanoparticle geometry and capping agent due to different synthesis methods play secondary roles.

Similar to $\mathrm{Cl}^{-}$, the addition of $\mathrm{Ag}^{+}, \mathrm{Mg}^{2+}$ or $\mathrm{Ca}^{2+}$ to cit-AgNPs mediates the electronic coupling between citrate and the metallic silver surface (Figure 5B). Indeed, right after adding $1 \mathrm{mM} \mathrm{AgNO}_{3}$ or $10^{-4} \mathrm{M} \mathrm{MgSO}_{4}$ or $\mathrm{Ca}\left(\mathrm{NO}_{3}\right)_{2}$, the SERS effect is turned on and intense SERS spectra of citrate can be observed (Figure 5B) [30]. The main SERS bands of citrate in Figure 5B appear shifted towards lower wavenumbers compared to the Raman bands, indicating a chemical interaction between the citrate molecules and the silver surface.
Thus, the SERS bands of citrate previously reported by us $[31,32]$ during the laser induced synthesis of SERS-active silver spots using silver nitrate-citrate mixtures can easily be explained by the presence of excess of $\mathrm{Ag}^{+}$in the solution, which induces the chemisorption of citrate onto the metal silver surface.

Nevertheless, we could not obtain the SERS spectrum of citrate when using silver colloids which contain chloride (hya-AgNPs, Cl-AgNPs) or when using cit-AgNPs which were activated with $\mathrm{Cl}^{-}$in concentrations higher than $1 \mathrm{mM}$. This is because the citrate molecules cannot displace chloride ions from the AgNP surface due to the higher affinity for the surface of the chloride ions compared to citrate.

The affinity between anions and the activated AgNP surface [18] also explains why we did not observe any SERS signal from $\mathrm{NO}_{3}{ }^{-}$or $\mathrm{SO}_{4}{ }^{2-}$, which should exhibit characteristic bands at $1046 \mathrm{~cm}^{-1}$ and $980 \mathrm{~cm}^{-1}$, respectively [30,33] and are known to have a weak affinity for the silver surface. Therefore, $\mathrm{NO}_{3}{ }^{-}$ or $\mathrm{SO}_{4}{ }^{2-}$ cannot displace the more tightly adsorbed chloride or citrate molecules from the surface of the nanoparticles (Figure 5) [33].

Due to the specificity of the $\mathrm{Ca}^{2+}$ or $\mathrm{Cl}^{-}$SERS-active sites, the SERS spectrum of the anionic or cationic analyte in the same solution can be turned on sequentially. Thus, by activating the cit-AgNPs with $\mathrm{Ca}^{2+}$ or $\mathrm{Cl}^{-}$, the SERS spectrum of citrate or the spectrum of cationic dye was recorded sequentially from their mixture. Figures S5 and S6 in Supporting Information File 1 show the specificity of SERS detection of citrate and $\mathrm{R} 6 \mathrm{G}$ or crystal violet. A schematic representation of the specific chemisorption and SERS detection of citrate and crystal violet is shown in Supporting Information File 1, Figure S7.

Chloride-containing and chloride-free colloids can therefore lead to different SERS spectra. This feature was observed also for zwitterionic L-cysteine [34-36]. In view of the results presented here, L-cysteine is chemisorbed via its $\mathrm{COO}^{-}$group to the silver surface at $\mathrm{Ag}^{+}$SERS-active sites when using citAgNPs as a SERS substrate. However, in $\mathrm{Cl}^{-}$activated colloids, L-cysteine is chemisorbed by its $\mathrm{NH}_{3}{ }^{+}$group at $\mathrm{Cl}^{-}$SERS-active sites.

\section{Conclusion}

We described a simple protocol for synthesizing Cl-AgNPs with a mean diameter of $36 \mathrm{~nm}$ by photoconversion from $\mathrm{AgCl}$ precursor microparticles, in the absence of any organic reducing or capping agent. The as-synthesized $\mathrm{Cl}-\mathrm{AgNPs}$ are already highly SERS-active due to chemisorbed $\mathrm{Cl}^{-}$ions, a feature which 
renders the $\mathrm{Cl}-\mathrm{AgNPs}$ more advantageous compared to the as-synthesized hya-AgNPs or cit-AgNPs for acquiring SERS spectra of cationic molecules.

Comparative SERS experiments employing Cl-AgNPs, hyaAgNPs and cit-AgNPs colloids, which contain different amounts of $\mathrm{Cl}^{-}$ions, highlight fundamental aspects of SERS : the SERS effect is turned on only after the electronic coupling of the analyte to the silver surface at SERS-active sites.

The chemisorption of anionic species, like $\mathrm{Cl}^{-}$or citrate, is mediated by cations such as $\mathrm{Ag}^{+}, \mathrm{Mg}^{2+}$, or $\mathrm{Ca}^{2+}$. The electronic coupling of cationic analytes to the silver surface occurs at SERS-active sites on the AgNP surface formed by chemisorbed $\mathrm{Cl}^{-}$ions. Our preliminary studies indicate that chemisorbed $\mathrm{I}^{-}$and $\mathrm{Br}^{-}$anions also form SERS-active sites for cationic analytes.

The experiments presented in this study also suggest that the SERS-activation role of ions such as $\mathrm{Cl}^{-}, \mathrm{Ag}^{+}, \mathrm{Mg}^{2+}$ or $\mathrm{Ca}^{2+}$ is more important than the Raman enhancement due to nanoparticle aggregation. Thus, the generation of SERS-active sites is decisive for obtaining high intensity SERS spectra, this feature being more important than the size, geometry or the capping agent of the SERS substrate.

In summary, to fully exploit the enhancing property of colloidal AgNPs for SERS of cationic molecules, the silver colloid should contain $\mathrm{Cl}^{-}$anions in a concentration range between $10^{-3}-10^{-2} \mathrm{M}$ and $\mathrm{Mg}^{2+}$ or $\mathrm{Ca}^{2+}$ cations in concentrations between $10^{-4}-10^{-3} \mathrm{M}$. For the SERS detection of anionic analytes, chloride-free colloids should be considered.

\section{Experimental}

Analytical grade silver nitrate (puriss. p.a., Sigma-Aldrich), sodium chloride (Reag. Ph. Eur., Merck), sodium hydroxide $(50 \% \mathrm{w} / \mathrm{w}$ solution, Fluka Analytical), hydrogen peroxide (10\% w/w solution, AppliChem), sodium citrate dihydrate (99\%, Sigma-Aldrich), hydroxylamine hydrochloride (99\%, Sigma-Aldrich), magnesium sulfate (99\%, Sigma-Aldrich), calcium nitrate tetrahydrate (99\%, Sigma-Aldrich), potassium iodide (99\%, Sigma-Aldrich), crystal violet chloride (Reag. Ph. Eur., Merck), 9-aminoacridine hydrochloride monohydrate (for synthesis, Merck) and rhodamine 6G (99\%, Sigma-Aldrich) were dissolved in ultrapure water (Direct-Q 3 UV, Millipore, resistivity higher than $18 \mathrm{M} \Omega$ ).

\section{Synthesis of silver colloids}

The synthesis of all silver colloids used in this study is described below. After synthesis, all colloids were stored in ambient conditions.

\section{Silver nanoparticles obtained by citrate reduction (cit-AgNPs)}

To obtain the cit-AgNPs, $17 \mathrm{mg}$ of silver nitrate was solved in $98 \mathrm{~mL}$ ultrapure water and heated to boiling. Then, $0.020 \mathrm{~g}$ of citrate solved in $2 \mathrm{~mL}$ ultrapure water was added dropwise. The mixture was further boiled under vigorous stirring for $50 \mathrm{~min}$.

\section{Silver nanoparticles obtained by hydroxylamine hydrochloride reduction (hya-AgNPs)}

To prepare the hya-AgNPs, $17 \mathrm{mg}$ of silver nitrate were solved in $90 \mathrm{~mL}$ ultrapure water. In a separate container, $17 \mathrm{mg}$ of hydroxylamine hydrochloride and $1.2 \mathrm{~mL}$ of sodium hydroxide solution $(1 \%)$ were mixed in $10 \mathrm{~mL}$ ultrapure water. The silver colloid was obtained at room temperature by rapidly adding the hydroxylamine hydrochloride/sodium hydroxide mixture to the silver nitrate solution under vigorous stirring. The resulting colloid was further stirred for $5 \mathrm{~min}$ [3].

\section{Chloride-capped silver nanoparticles obtained by photoreduction (Cl-AgNPs)}

For the preparation of the new Cl-AgNPs, $40 \mathrm{~mL}$ of water was placed in a plastic beaker and the following reagents were added in the mentioned order: $400 \mu \mathrm{L}$ of a $1 \mathrm{M}$ sodium chloride solution, $180 \mu \mathrm{L}$ of a $1 \%$ sodium hydroxide solution, $200 \mu \mathrm{L}$ of a $10 \%$ hydrogen peroxide solution, $400 \mu \mathrm{L}$ of a $0.1 \mathrm{M}$ silver nitrate solution. The $\mathrm{pH}$ of the reaction mixture reduced from 10 to 7.5 during the synthesis.

The optimal amount of sodium chloride was determined from repeated synthesis experiments. Stable silver colloidal solutions were obtained by adding $1 \mathrm{M} \mathrm{NaCl}$ to the synthesis mixture at a volume between 300 and $500 \mu \mathrm{L}$.

After the addition of the reagents, the beaker was exposed to the light provided by a conventional desk lamp equipped with a commercial LED bulb (23 W, $6500 \mathrm{~K}$, cool white, $2452 \mathrm{~lm})$, under constant magnetic stirring (400 rotations/minute). Figure 1 shows a picture of the experimental setup and a picture of the resulting colloid.

For monitoring the photosynthesis of Cl-AgNPs, aliquots of $100 \mu \mathrm{L}$ were taken from the reaction mixture at sequential time points during the synthesis reaction, diluted 10-fold, and analyzed by UV-vis absorption, as shown in Figure 2.

Prior to the electron microscopy analysis, a drop of colloidal solution was deposited and dried on a copper grid coated by a thin carbon film. The measurements were carried out using a Hitachi HD-2700 scanning transmission electron microscope (STEM), equipped with a cold field emission gun, working at an acceleration voltage of $200 \mathrm{kV}$. 
The absorption spectra were recorded with a Jasco V-630 $\mathrm{UV}$-vis spectrometer at $1 \mathrm{~nm}$ spectral resolution.

Raman and SERS spectra were obtained with a Renishaw InVia Raman microscope, equipped with a Nd:YAG frequencydoubled laser emitting at $532 \mathrm{~nm}$ and a laser power of $\approx 60 \mathrm{~mW}$ on the sample. For each measurement, $10 \mu \mathrm{L}$ of the sample was placed on a microscope slide that was covered with aluminum foil. The laser light was focused on the drop using a $5 \times$ objective (NA 0.12). The spectra were recorded by averaging four acquisitions of $4 \mathrm{sec}$ each.

\section{Supporting Information}

\section{Supporting Information File 1}

Additional experimental data.

[https://www.beilstein-journals.org/bjnano/content/ supplementary/2190-4286-9-208-S1.pdf]

\section{Acknowledgements}

This work was supported by a grant of the Romanian Ministry of Research and Innovation, CCCDI-UEFISCDI, project number PN-III-P1-1.2-PCCDI-2017-0056, within PNCDI III. Sebastian Porav from INCDTIM Cluj-Napoca is highly acknowledged for the electron microscopy measurements. Dr. Zoltán Bálint and Dr. Vasile Coman are highly acknowledged for reading the manuscript and providing useful suggestions.

\section{ORCID ${ }^{\circledR}$ iDs}

Nicolae Leopold - https://orcid.org/0000-0002-2174-8064

\section{References}

1. Lee, P. C.; Meisel, D. J. Phys. Chem. 1982, 86, 3391-3395. doi:10.1021/j100214a025

2. Creighton, J. A.; Blatchford, C. G.; Albrecht, M. G. J. Chem. Soc., Faraday Trans. 2 1979, 75, 790-798. doi:10.1039/f29797500790

3. Leopold, N.; Lendl, B. J. Phys. Chem. B 2003, 107, 5723-5727. doi:10.1021/jp027460u

4. Gao, X.; Wan, C.; He, T.; Li, J.; Xin, H.; Liu, F.-c. Chem. Phys. Lett 1984, 112, 465-468. doi:10.1016/0009-2614(84)85570-0

5. Ahern, A. M.; Garrell, R. L. Anal. Chem. 1987, 59, 2813-2816. doi:10.1021/ac00150a020

6. Hassoun, M.; Schie, I. W.; Tolstik, T.; Stanca, S. E.; Krafft, C.; Popp, J. Beilstein J. Nanotechnol. 2017, 8, 1183-1190. doi:10.3762/bjnano.8.120

7. Maruyama, Y.; Futamata, M. Chem. Phys. Lett. 2007, 448, 93-98. doi:10.1016/j.cplett.2007.09.056

8. Kneipp, K.; Kneipp, H.; Itzkan, I.; Dasari, R. R.; Feld, M. S. Chem. Rev. 1999, 99, 2957-2976. doi:10.1021/cr980133r

9. Stiles, P. L.; Dieringer, J. A.; Shah, N. C.; Van Duyne, R. P. Annu. Rev. Anal. Chem. 2008, 1, 601-626. doi:10.1146/annurev.anchem.1.031207.112814
10. Farcau, C.; Astilean, S. J. Phys. Chem. C 2010, 114, 11717-11722. doi:10.1021/jp100861w

11. Moskovits, M. J. Raman Spectrosc. 2005, 36, 485-496. doi:10.1002/jrs. 1362

12. Otto, A.; Mrozek, I.; Grabhorn, H.; Akemann, W. J. Phys.: Condens. Matter 1992, 4, 1143-1212. doi:10.1088/0953-8984/4/5/001

13. Otto, A.; Futamata, M. Electronic Mechanisms of SERS. In Surface-Enhanced Raman Scattering: Physics and Applications; Kneipp, K.; Moskovits, M.; Kneipp, H., Eds.; Springer Berlin Heidelberg: Berlin, Heidelberg, 2006; pp 147-182. doi:10.1007/3-540-33567-6_8

14. Lopez-Ramirez, M. R.; Garcia-Ramos, J. V.; Otero, J. C.; Castro, J. L.; Sanchez-Cortes, S. Chem. Phys. Lett. 2007, 446, 380-384. doi:10.1016/j.cplett.2007.08.069

15. Billmann, J.; Kovacs, G.; Otto, A. Surf. Sci. 1980, 92, 153-173. doi:10.1016/0039-6028(80)90250-2

16. Vo-Dinh, T. Surface-Enhanced Raman Scattering. In Photonic Probes of Surfaces; Halevi, P., Ed.; Elsevier: Amsterdam, 1995; pp 65-95. doi:10.1016/B978-0-444-82198-0.50009-6

17. Otto, A.; Bruckbauer, A.; Chen, Y. X. J. Mol. Struct. 2003, 661-662, 501-514. doi:10.1016/j.molstruc.2003.07.026

18. Muniz-Miranda, M.; Sbrana, G. J. Raman Spectrosc. 1996, 27, 105-110. doi:10.1002/(SICI)1097-4555(199602)27:2<105::AID-JRS933>3.0.CO; 2-L

19. Sato-Berrú, R.; Redón, R.; Vázquez-Olmos, A.; Saniger, J. M. J. Raman Spectrosc. 2009, 40, 376-380. doi:10.1002/jrs.2135

20. Huang, H. H.; Ni, X. P.; Loy, G. L.; Chew, C. H.; Tan, K. L.; Loh, F. C.; Deng, J. F.; Xu, G. Q. Langmuir 1996, 12, 909-912. doi:10.1021/la950435d

21. Xu, G.-n.; Qiao, X.-I.; Quu, X.-I.; Chen, J.-g. Colloids Surf., A 2008, 320, 222-226. doi:10.1016/j.colsurfa.2008.01.056

22. Mukherjee, P.; Katarya, A.; McMaster, N.; Gonzalez, M. A.; Bhattacharya, R. Int. J. Green Nanotechnol. 2011, 3, 134-139. doi:10.1080/19430892.2011.574930

23. Wang, G.; Nishio, T.; Sato, M.; Ishikawa, A.; Nambara, K.; Nagakawa, K.; Matsuo, Y.; Niikura, K.; Ijiro, K. Chem. Commun. 2011, 47, 9426-9428. doi:10.1039/c1cc13385c

24. Wang, G.; Mitomo, H.; Matsuo, Y.; Niikura, K.; Maeda, M.; ljiro, K. J. Colloid Interface Sci. 2015, 452, 224-234. doi:10.1016/j.jcis.2015.04.033

25. Wang, P.; Huang, B.; Lou, Z.; Zhang, X.; Qin, X.; Dai, Y.; Zheng, Z.; Wang, X. Chem. - Eur. J. 2010, 16, 538-544. doi:10.1002/chem.200901954

26. Rasulov, B. A.; Rozi, P.; Pattaeva, M. A.; Yili, A.; Aisa, H. A. Materials 2016, 9, No. 528. doi:10.3390/ma9070528

27. Wang, P.; Huang, B.; Qin, X.; Zhang, X.; Dai, Y.; Wei, J.; Whangbo, M.-H. Angew. Chem., Int. Ed. 2008, 47, 7931-7933. doi:10.1002/anie.200802483

28. Futamata, M.; Maruyama, Y. Anal. Bioanal. Chem. 2007, 388, 89-102. doi:10.1007/s00216-007-1183-5

29. Doering, W. E.; Nie, S. J. Phys. Chem. B 2002, 106, 311-317. doi:10.1021/jp011730b

30. Bell, S. E. J.; Sirimuthu, N. M. S. J. Phys. Chem. A 2005, 109, 7405-7410. doi:10.1021/jp052184f

31. Leopold, N.; Lendl, B. Anal. Bioanal. Chem. 2010, 396, 2341-2348. doi:10.1007/s00216-010-3468-3 
32. Herman, K.; Szabó, L.; Leopold, L. F.; Chiş, V.; Leopold, N. Anal. Bioanal. Chem. 2011, 400, 815-820. doi:10.1007/s00216-011-4798-5

33. Stewart, A.; Murray, S.; Bell, S. E. J. Analyst 2015, 140, 2988-2994. doi:10.1039/C4AN02305F

34. Jing, C.; Fang, Y. Chem. Phys. 2007, 332, 27-32. doi:10.1016/j.chemphys.2006.11.019

35. Diaz Fleming, G.; Finnerty, J. J.; Campos-Vallette, M.; Célis, F.; Aliaga, A. E.; Fredes, C.; Koch, R. J. Raman Spectrosc. 2009, 40, 632-638. doi:10.1002/jrs.2175

36. López-Tobar, E.; Hernández, B.; Ghomi, M.; Sanchez-Cortes, S. J. Phys. Chem. C 2013, 117, 1531-1537. doi:10.1021/jp3112606

\section{License and Terms}

This is an Open Access article under the terms of the Creative Commons Attribution License

(http://creativecommons.org/licenses/by/4.0). Please note that the reuse, redistribution and reproduction in particular requires that the authors and source are credited.

The license is subject to the Beilstein Journal of Nanotechnology terms and conditions:

(https://www.beilstein-journals.org/bjnano)

The definitive version of this article is the electronic one which can be found at: doi:10.3762/bjnano.9.208 